\title{
Does a Relationship Exist Between Posterior Vitreous Detachment, Macular Thickness and Foveal Avascular Zone Dimensions in Myopic Eyes?
}

Jothi Balaji Janarthanam

Sankara Nethralaya, Medical Research Foundation, Chennai, India

Arpit Agarwal

Indian Institute of Technology Kanpur

Rajiv Raman ( $\nabla$ rajivpgraman@gmail.com )

Sankara Nethralaya, Medical Research Foundation, Chennai, India

Lakshminarayanan Vasudevan

University of Waterloo

\section{Research Article}

Keywords: Myopia, Posterior vitreous detachment, Foveal avascular zone, OCT, Macular thickness

Posted Date: March 11th, 2021

DOI: https://doi.org/10.21203/rs.3.rs-272664/v1

License: (c) (i) This work is licensed under a Creative Commons Attribution 4.0 International License.

Read Full License 

Thickness and Foveal Avascular Zone Dimensions in Myopic Eyes?

3

4 J. Jothi Balaji ${ }^{1}$, Arpit Agarwal ${ }^{2}$, Rajiv Raman ${ }^{1 *}$, Vasudevan Lakshminarayanan ${ }^{3}$

$5 \quad{ }^{1}$ Sankara Nethralaya, Medical Research Foundation, Chennai, India

$6 \quad{ }^{2}$ Department of Chemical Engineering, Indian Institute of Technology, Kanpur, India

$7{ }^{3}$ Theoretical and Experimental Epistemology Lab, School of Optometry and Vision Science,

8 University of Waterloo, Waterloo, Ontario, Canada

9

10 *Corresponding author: Rajiv Raman

11 Shri Bhagwan Mahavir Vitreoretinal Services, Sankara Nethralaya, Medical Research

12 Foundation, 18, College Road, Nungambakkam, Chennai, India. Pin: 600006

13 Tel: $+91-44-42271500$

14 Fax: +91-44-2825 4180

15 Email: rajivpgraman@gmail.com

16

17 
Abstract

Background: The posterior vitreous detachment (PVD) is a separation of the posterior vitreous cortex from the internal limiting membrane of the retina. The PVD induces several potentially serious pathologic events at the vitreoretinal interface. The aim of the study is to determine if relationships exist between PVD, macular thickness (MT) and the foveal avascular zone (FAZ) in myopic eyes.

Methods: This is a retrospective case study of 63 myopic subjects who underwent comprehensive eye examination including the optical coherence tomography angiography (OCTA) between January 1 and Jun 30, 2019. The spherical equivalent (SE) was calculated using the manifest refraction. The myopia grouping was based on a severity scale, namely mild, moderate, high and very high using standard refractive error classifications. The PVD classification was based on optical coherence tomography (OCT) images. The status of the PVD and MT were evaluated with Macular Cube $200 \times 200$ images and the FAZ was imaged with an OCTA, Angioplex. The MT and FAZ dimensions were calculated using a custom algorithm.

Results: A total of 114 myopic eyes subjects had median (range) age of 26.00 (22.00 28.00) years. Of this cohort, females comprised $62.3 \%$ of the dataset, and the right eyes were $50.00 \%$. In this population, 10 eyes had no PVD in any quadrant, and 73 eyes had incomplete PVD in all four quadrants. The inferior quadrant had the maximum number of PVD cases and the nasal quadrant had the least number of cases. High myopic eyes exhibited significantly increased low foveal volume $(p=0.000)$. The inferior part of para- and perimacular area showed a significant thinning in very high myopic eyes $(p=<0.05)$. A statistically significant alteration of FAZ circularity index found in very high myopic eyes $(p=0.002)$. 
42 Conclusion: In high and very high myopic eyes, an increasing trend of partial PVD is

43

44

45

seen. The most commonly involved location was the inferior quadrant. A significant alteration in foveal volume and circularity index of the FAZ is seen in high and very high myopic eyes.

Keywords: Myopia, Posterior vitreous detachment, Foveal avascular zone, OCT, Macular thickness

\section{Background}

Liquefaction of the vitreous gel which is attached to the internal limiting membrane of the retina [1-3] or spontaneous posterior vitreous detachment (PVD) from the retina is considered to be a part of normal aging process $[1,4]$. The onset of PVD is usually reported around the fifth decade of life or later in normal [4]. The shallow PVD not only helps to predict the prognosis and also determines the indication for vitreoretinal surgery in many vitreoretinal conditions [5]. The usual slit lamp examination with a +90D lens fails to detect shallow PVDs [5] Currently, the spectral-domain optical coherence tomography (SD-OCT) is commonly used [2] to image the structural relationship of the vitreomacular interface $[3,6]$. The SD-OCT method not only helps to qualitatively diagnose different stages of disease but can also be used to quantify the PVD stages.

The increasing prevalence of myopia worldwide $[7,8]$ can lead to potentially blinding ocular complications especially in the posterior part of the eyes in pathological myopes [7]. Usually the pathological myopic complications are bilateral [9]. Myopes are reported to have long axial length especially in cases of high myopia $(>26.5 \mathrm{~mm})$ due to scleral ectasia $[7,8,10]$. Rhegmatogenous retinal detachment has a higher incidence in axial myopic subjects $[4,11]$. Foveal retinoschisis and posterior staphyloma are also reported in high myopia [4]. Bond- 
89

Taylor et al. [12] reported the PVD prevalence in normal individuals was $24 \%$ among subjects aged between $50-59$ and increases to $89 \%$ in the $9^{\text {th }}$ decade of life.

However, in high myopes, the onset of PVD has been reported to occur much earlier than nonmyopic or low-moderate myopic eyes [4,13]. For example, Akiba [11] using a slit-lamp method reported that the onset of PVD in high myopes was in the third decade of life. Similarly, Itakura et al. [4] also reported that in high myopes (worse than -8.00D) PVD could occur as early as the third decade of life.

Many authors have hypothesized that retinal changes occur as a result of mechanical stretching due to axial elongation in myopia $[7,8,14]$. During axial stretching the traction of the vitreous on the fovea in myopic eyes causes structural changes [7]. Chung et al. [15] reported no significant foveal thickness changes with the axial length up to an axial length of $25.5 \mathrm{~mm}-26.00 \mathrm{~mm}$. In high myopic eyes (SE worse than $-8.00 \mathrm{D}$ or $\mathrm{AXL} \geq 26.00 \mathrm{~mm}$ ), a significantly thicker fovea was found with a steep foveal slope. $[7,16]$

Gomaa and Abouhussein [17] reported that retinal morphological changes are common in high myopic eyes and recommended that OCT based investigation should be made routinely. Ito et al. [18] reported close correlations between the degree of PVD and the stages of idiopathic macular hole. Xu et al. [19] classified the vitreomacular interface diseases and its sub-classifications based on vitreomacular adhesion and vitreomacular traction. Hence foveal morphological changes are expected in high myopia, especially in the superficial retinal layers [20] and blood vessels.

To the best of our knowledge there are no reports investigating the relationship between PVD, central macular thickness (CRT) and foveal microvascular zone (FAZ) dimensions in myopic eyes. This is the subject of this study. 


\section{Subject}

94 The electronic medical records (which included OCTA) of all myopic subjects who visited a

95 tertiary eye care hospital's refractive surgery clinic, in Chennai, India between January 1 and

96 Jun 30, 2019 were reviewed. A total of sixty-three subjects (114 eyes) were included in the

97 study. Myopia was classified into four groups based on spherical equivalent (SE) [21], namely

98 Iow myopia (SE between -0.12 and $-3.00 \mathrm{D}$ ), moderate myopia (SE between -3.12 and -5.00

99 D), high myopia (SE between -5.12 and $-10.00 \mathrm{D}$ ) and very high myopia (SE worse than - 10.00

D). The SE was calculated using the manifest refraction during the ophthalmic examination.

101 The exclusion criteria were any prior history or clinical evidence of retinal or systemic disease.

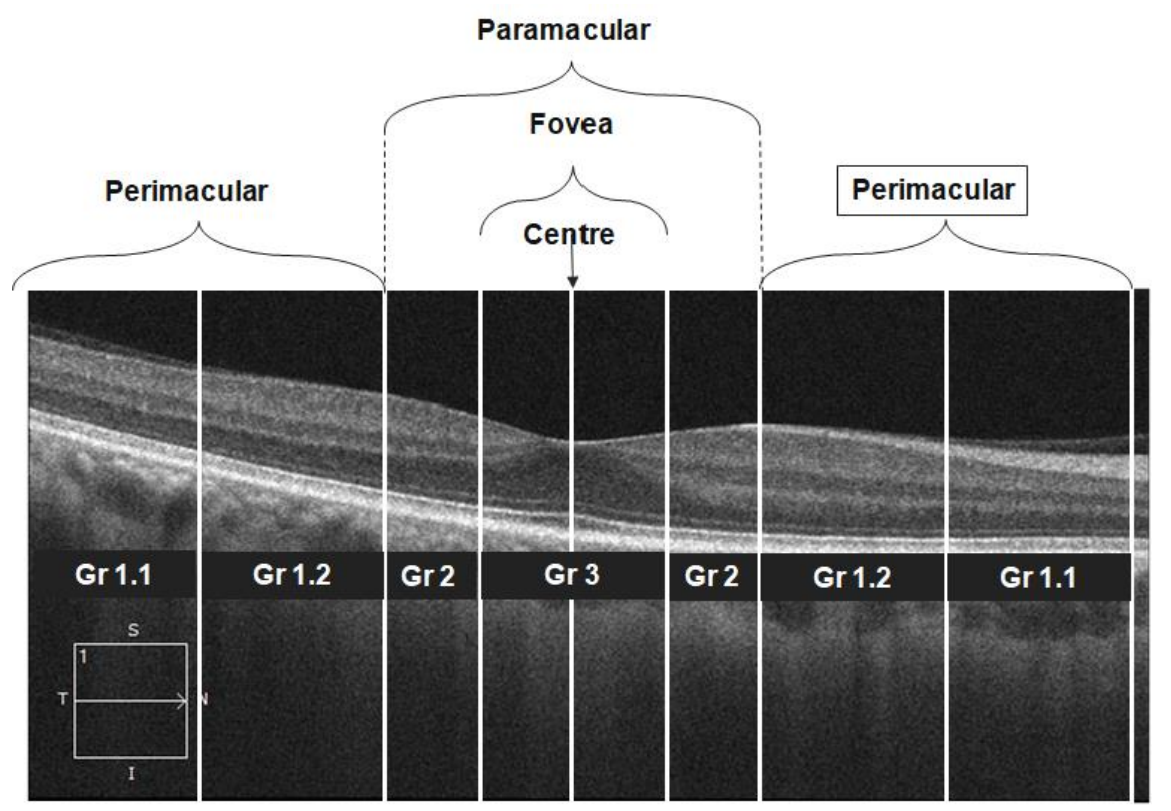

Figure 1: An example and a schematic diagram of a SD-OCT horizontal radial scan image. The PVD classification based on the PVD location. The above picture shows the PVD extended till parimacular area with grade 1.2. 

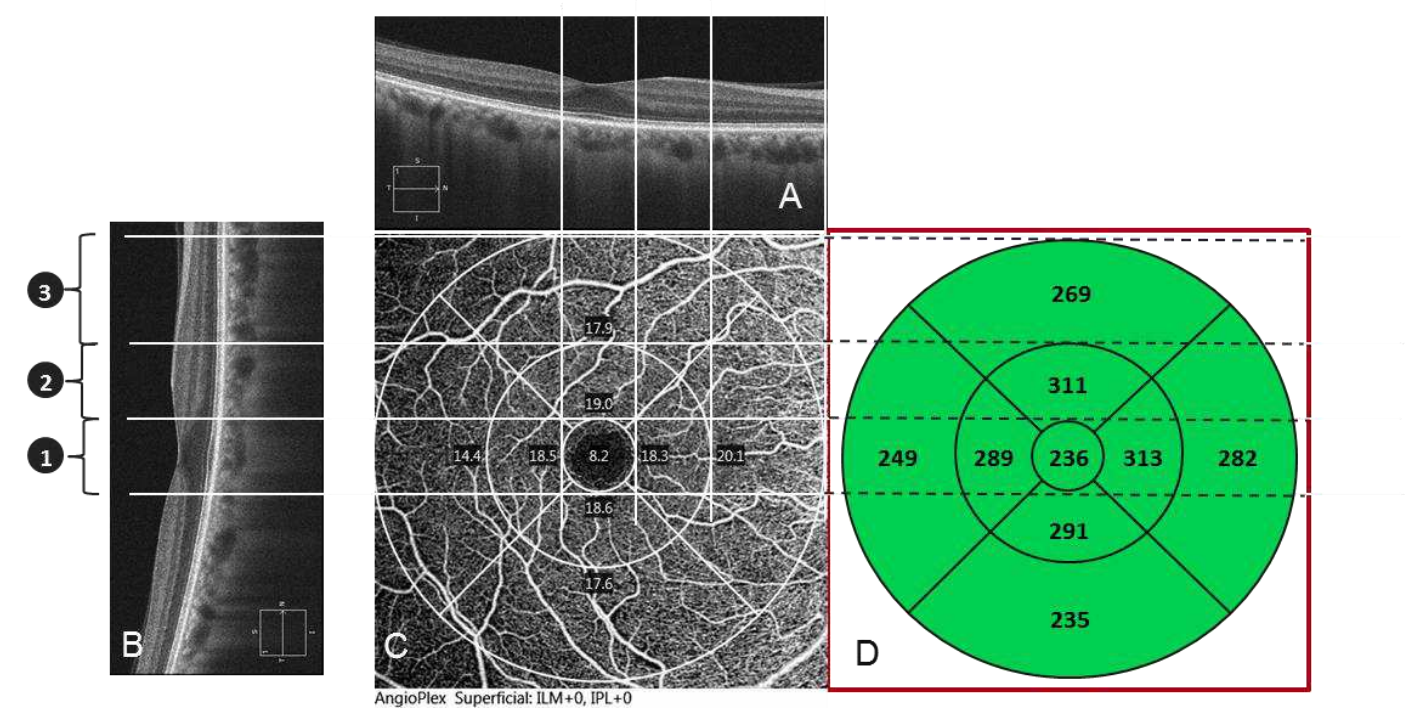

Figure 2: A relationship between PVD location and the corresponding location macular thickness and angioplex image using the ETDRS method. A \& B) a radial scan of a horizontal and vertical scan. The numbers 1,2 \& 3 indicating the macular anatomical structures 1) fovea, 2) paramacular and 3) perimacular area. C) An en-face image of macular and FAZ with ETDRS segmentation D) the ETDRS macular thickness (in microns) to the corresponding area of the fovea, para, and peri macular regions.

\section{Central macular thickness and Foveal microvascular dimensions}

Both central macular thickness (Macular cube $200 \times 200$ ) and foveal microvascular dimensions

(Angioplex) were imaged using optical coherence tomography angiography (OCTA; Cirrus

inbuilt in the device. The foveal microvascular dimensions were calculated using a custom$\mathrm{mm}$ (vertical $\mathrm{x}$ horizontal) window axial scans in the macular region. The macular region was divided into 9 quadrants with 3 concentric rings [7] (The central $1.0 \mathrm{~mm}$ diameter ring represents the fovea. the second $2.0 \mathrm{~mm}$ diameter ring represents the para-macular region and the outer most $3.0 \mathrm{~mm}$ ring represents the peri-macula; Figure 2). The macular analysis. 


\section{PVD Classification}

120 A single clinical expert (RRN) classified the PVD based on the OCT radial images of both vertical

121 and horizontal. Since most of the cases had very early PVD, the method for PVD classification

122 given by Itakura et al [4] was modified. Figure 1 shows the stages of PVDs and its classification.

123 If no PVD was seen within the $6 \mathrm{~mm}$ scan it was graded as Grade 0. The Grade 1 PVD is a

124 perimacular area attached PVD, the Grade 2 is the PVD attached to the paramacular area.

125 Grade 3 is the foveally attached PVD. Grade 1 covers a larger area and hence it was further

126 sub-classified to 1.1 and 1.2 based on the PDV location (Figure 1). The PVD was graded for all

127 four quadrants (superior, nasal, inferior and temporal).

128

\section{Statistical analysis}

130 All statistical analyses were performed using the SPSS version 20 (SPSS Inc, Chicago, Illinois,

131 USA). The normal distribution of quantitative variables was checked using the Kolmogorov-

132 Smirnov test. A one-way analysis of variance (ANOVA) and Kruskal Wallis analysis was

133 performed for comparison between PVD groups. A two-sided unpaired student t-test was

134 used to compare the various parameters of the sub-group myopic groups. The $p$-value $<0.05$

135 was considered significant for all the statistical tests.

\section{Results}

138 Out of the total of 114 eyes, 43 were male (37.72\%) and 71 were female (62.28\%). The 139 median \pm interquartile range (IQR) of age, best corrected visual acuity (BCVA) and SE of this 140 population were $26.00(22.00-28.00)$ years, $0.00(0.00-0.00) \log M A R$ and -5.56 D $(-3.09 \mathrm{D}$ $141-8.06 \mathrm{D})$ respectively. The median (IQR) of CMT, FAZ area and macular cube volume (MCV) 142 were $248.00(235.00-260.00) \mu \mathrm{m}, 0.27(0.20-0.34) \mathrm{mm}^{2}$ and $9.80(9.60-10.10)$ for the full 143 sample. Table 1 shows the study sample demographic and clinical characteristics and their 144 sub-groups based on PVD stages. 


\begin{tabular}{lccc}
\hline & Age (years) & SE (D) & BCVA (LogMAR) \\
\hline Low myopia & $23.0(21.0-27.0)$ & $-2.25(-1.37$ to -2.50$)$ & $0.0(0.0-0.0)$ \\
Moderate myopia & $26.0(23.0-31.0)$ & $-4.25(-3.50$ to -4.50$)$ & $0.0(0.0-0.0)$ \\
High myopia & $26.0(23.0-28.0)$ & $-6.44(-6.25$ to -7.75$)$ & $0.0(0.0-0.0)$ \\
Very High myopia & $27.0(22.5-30.0)$ & $-14.25(-11.25$ to -18.06$)$ & $0.0(0.0-0.1)$ \\
Overall myopia & $26.0(22.0-28.0)$ & $-5.56(-3.09$ to -8.06$)$ & $0.0(0.0-0.0)$ \\
p-Value & $0.568^{*}$ & $0.000^{* *}$ & $0.000^{* *}$ \\
\hline *One-Way ANOVA, **Kruskal Wallis, SE: Mean spherical equivalent, BCVA: Best corrected visual acuity
\end{tabular}
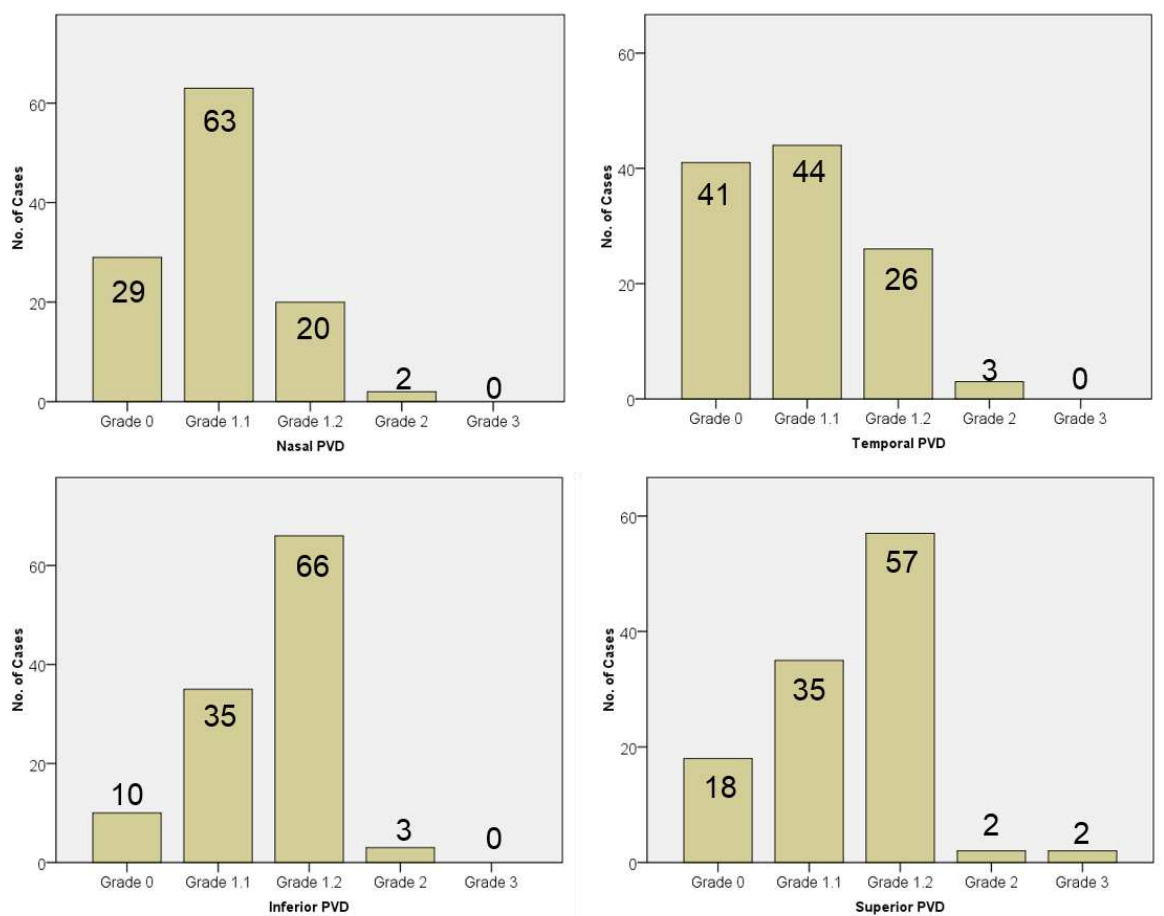

Figure 3: Distribution of PVD in all four quadrants.

150 The stage of the PVD was classified using the predefined PVD grading scheme (Figure 1). Out

151 of 114 myopic eyes, 10 eyes (8.77 \%) had no PVD in any of the quadrants, 31 eyes (27.19\%)

152 had PVD in one or more than one quadrant, and 73 eyes (64.04 \%) had PVD in all four

153 quadrants. The figure 3 shows the frequency distribution of PVD in all four quadrants. The

154 inferior quadrant PVD was common and PVD in the nasal quadrant was least common. Figure

1554 \& 5 show the quadrants wise changes in macular thickness in different degrees of myopia. 


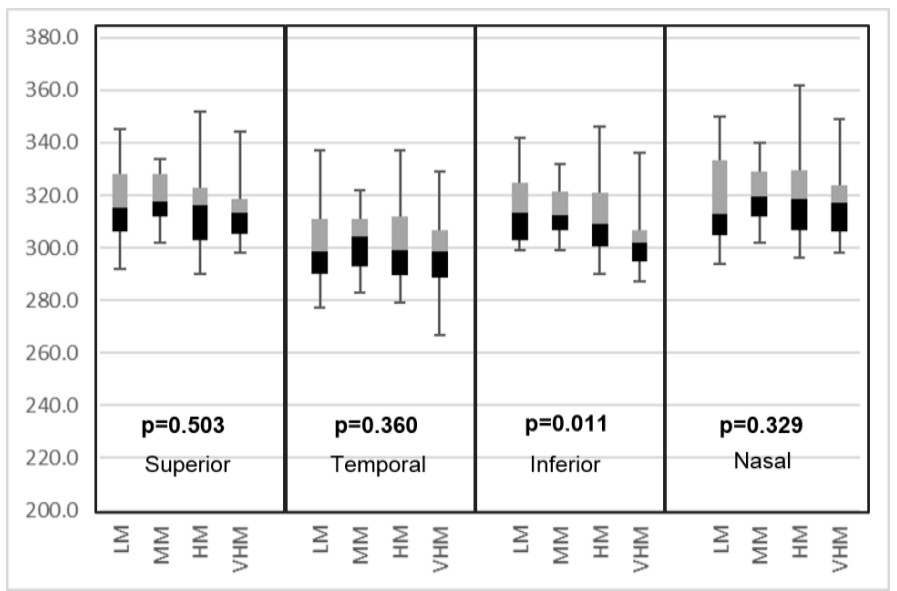

Figure 4: A box-plot between para-macular thickness in four quadrants and degree of myopia

LM: Low myopia, MM: Moderate myopia, HM: High myopia VHM: Very high myopia

\begin{tabular}{|c|c|c|c|c|}
\hline 2750 & $p=0.335$ & $p=0.009$ & $p=0.002$ & $p=0.802$ \\
\hline 350.0 & & & & \\
\hline 325.0 & & & & \\
\hline 300.0 & & & & \\
\hline 275.0 & & & & 1 \\
\hline 250.0 & & & & \\
\hline \multirow{2}{*}{200.0} & $\begin{array}{l}\text { Superior } \\
\text { Quadrant }\end{array}$ & $\begin{array}{l}\text { Temporal } \\
\text { Quadrant }\end{array}$ & $\begin{array}{c}\text { Inferior } \\
\text { Quadrant }\end{array}$ & $\begin{array}{c}\text { Nasal } \\
\text { Quadrant }\end{array}$ \\
\hline & $\sum \sum \sum \sum$ & $\sum \sum \sum \sum$ & $\sum \sum \sum \sum$ & $\sum \sum \sum \sum$ \\
\hline
\end{tabular}

Figure 5: A box-plot of Peri-macular thickness in all the four quadrants and degree of myopia 
Table 2: FAZ and Foveal dimensions for different degrees of myopia

\begin{tabular}{|c|c|c|c|c|c|c|}
\hline \multirow[b]{2}{*}{ Myopia } & \multicolumn{3}{|c|}{ FAZ dimension } & \multicolumn{3}{|c|}{ Foveal characteristics } \\
\hline & $\begin{array}{c}\text { Area } \\
\left(\mathrm{mm}^{2}\right)\end{array}$ & $\begin{array}{l}\text { Perimeter } \\
(\mathrm{mm})\end{array}$ & Circularity & $\begin{array}{c}\text { Thickness } \\
(\mu \mathrm{m})\end{array}$ & $\begin{array}{l}\text { Volume } \\
\left(\mathrm{mm}^{3}\right)\end{array}$ & $\begin{array}{l}\text { Vessel } \\
\text { Density }\end{array}$ \\
\hline Low & $\begin{array}{c}0.26 \\
(0.21-0.34)\end{array}$ & $\begin{array}{c}2.02 \\
(1.85-2.45)\end{array}$ & $\begin{array}{c}0.79 \\
(0.71-0.81)\end{array}$ & $\begin{array}{c}245.0 \\
(230.0-254.0)\end{array}$ & $\begin{array}{c}9.80 \\
(9.60-10.30)\end{array}$ & $\begin{array}{c}9.50 \\
(6.90-11.40)\end{array}$ \\
\hline Moderate & $\begin{array}{c}0.28 \\
(0.20-0.34)\end{array}$ & $\begin{array}{c}2.17 \\
(1.87-2.30)\end{array}$ & $\begin{array}{c}0.75 \\
(0.73-0.78)\end{array}$ & $\begin{array}{c}251.0 \\
(238.0-260.0)\end{array}$ & $\begin{array}{c}9.90 \\
(9.80-10.00)\end{array}$ & $\begin{array}{c}9.90 \\
(7.60-10.80)\end{array}$ \\
\hline High & $\begin{array}{c}0.29 \\
(0.18-0.37)\end{array}$ & $\begin{array}{c}2.20 \\
(1.78-2.47)\end{array}$ & $\begin{array}{c}0.76 \\
(0.67-0.77)\end{array}$ & $\begin{array}{c}249.0 \\
(235.0-258.0)\end{array}$ & $\begin{array}{c}9.90 \\
(9.60-10.30)\end{array}$ & $\begin{array}{c}8.85 \\
(7.00-10.80)\end{array}$ \\
\hline Severe & $\begin{array}{c}0.27 \\
(0.21-0.31)\end{array}$ & $\begin{array}{c}2.16 \\
(1.85-2.47)\end{array}$ & $\begin{array}{c}0.70 \\
(0.61-0.74)\end{array}$ & $\begin{array}{c}249.0 \\
(238.0-267.0)\end{array}$ & $\begin{array}{c}9.50 \\
(9.10-9.80)\end{array}$ & $\begin{array}{c}7.90 \\
(4.70-10.45)\end{array}$ \\
\hline Total & $\begin{array}{c}0.27 \\
(0.20-0.34)\end{array}$ & $\begin{array}{c}2.16 \\
(1.81-2.43)\end{array}$ & $\begin{array}{c}0.75 \\
(0.69-0.79)\end{array}$ & $\begin{array}{c}248.0 \\
(235.0-260.0)\end{array}$ & $\begin{array}{c}9.80 \\
(9.60-10.10)\end{array}$ & $\begin{array}{c}9.10 \\
(6.80-11.10)\end{array}$ \\
\hline p-Value & $0.731^{*}$ & $0.774 *$ & $0.002 * *$ & $0.131 *$ & $0.000 *$ & $0.195 *$ \\
\hline
\end{tabular}

167

168 Table 2 shows an alteration in FAZ dimension and foveal characteristics in different degree of 169 myopia. Only the FAZ circularity $(p=0.00)$ and foveal volume $(p=<0.00)$ exhibited significantly 170 alteration as the degree of myopia increases.

171

\section{Table 3: A cross correlation table between PVD location and degree of myopia}

\begin{tabular}{|c|c|c|c|c|c|}
\hline & \multicolumn{4}{|c|}{ Myopia } & \\
\hline & Low & Moderate & High & Severe & Average \\
\hline Superior & 78.60 & 83.30 & 89.50 & 83.30 & 83.68 \\
\hline Temporal & 50.00 & 83.30 & 63.30 & 62.50 & 64.78 \\
\hline Inferior & 82.10 & 95.80 & 97.40 & 87.50 & 90.70 \\
\hline Nasal & 64.30 & 79.30 & 81.30 & 70.80 & 73.93 \\
\hline Average & 68.75 & 85.43 & 82.88 & 76.03 & \\
\hline
\end{tabular}

175 Table 3 shows a cross correlation table between PVD location and degree of myopia. We

176 found an asymmetry in the PVD location. The results suggest an increasing trend of PVD as

177 the degree of myopia increases with a location asymmetry. In low myopia the temporal

178 quadrant showed the least PVD (50.0\%) whereas high myopic eyes exhibited the most PVD in

179 the inferior quadrant (97.40\%). Figure 6 \& 7 show the relationship between paramacular and 180 peri-macular vessel density changes in various stages of PVD. In the paramacular area, the 
181 inferior and nasal quadrants showed a significantly decreased vessel density $(p=<0.05)$.

182 However, in the peri-macular area, the nasal quadrant alone showed significant loss of vessel

183 density. Table 4 shows the correlation between myopia (SE) verses macular thickness and macular vessel density different ETDRS quadrants. A significant negative correlation was found between myopia and foveal thickness. However, a weak positive correlation was seen with inferior para-macular region and overall peri-macular thickness.

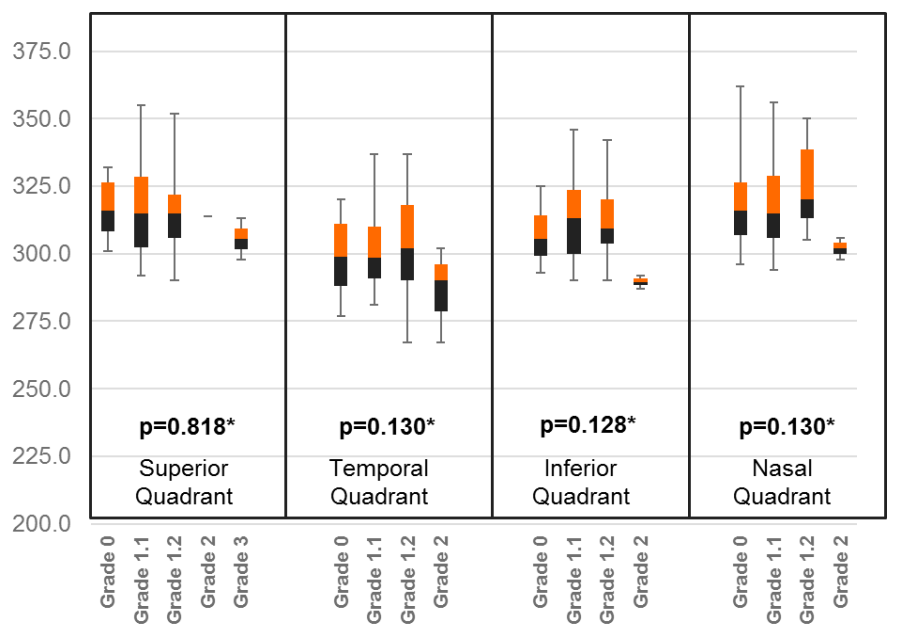

Figure 6: Relationship between paramacular vessel density \& PVD stages

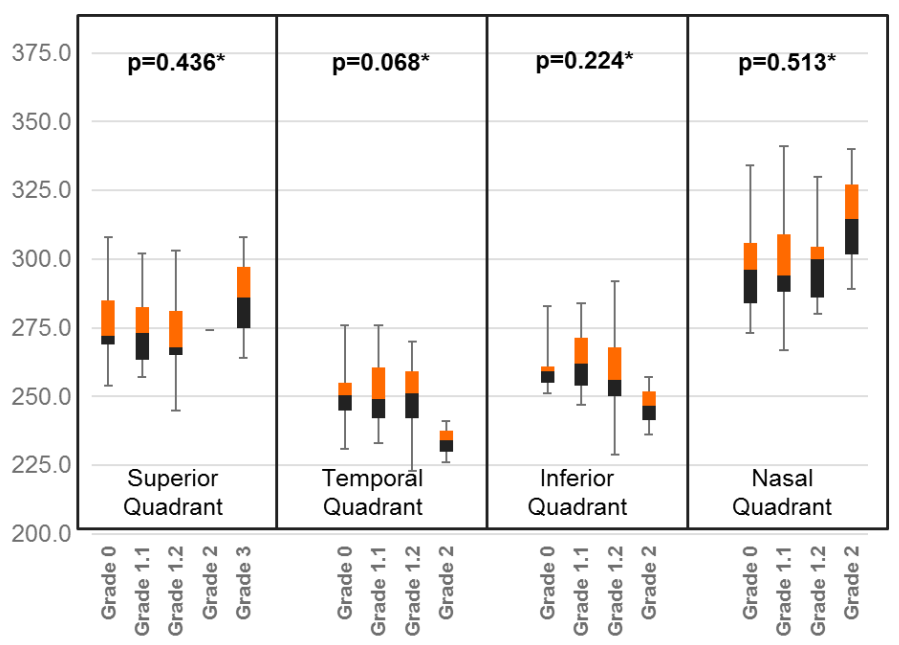

Figure 7: Relationship between peri-macular vessel density \& PVD stages 
Table 4: Correlation between degree of myopia and macular dimensions.

\begin{tabular}{|lcccc|}
\hline & \multicolumn{2}{c}{ Thickness $(\boldsymbol{\mu m})$} & \multicolumn{2}{c|}{ Vessel density } \\
& $\mathrm{r}^{*}$ & $\mathbf{p}$ Value & $\mathbf{r}^{*}$ & $\mathrm{p}$ Value \\
\hline Foveal area & $-\mathbf{0 . 2 6 1}$ & $\mathbf{0 . 0 0 7}$ & 0.253 & 0.066 \\
Average para-macular & 0.097 & 0.330 & $\mathbf{0 . 5 1 9}$ & $<0.000$ \\
Superior para -macular & 0.065 & 0.514 & $\mathbf{0 . 5 0 0}$ & $<\mathbf{0 . 0 0 0}$ \\
Temporal para-macular & 0.094 & 0.344 & $\mathbf{0 . 4 4 8}$ & $<\mathbf{0 . 0 0 0}$ \\
Inferior para-macular & $\mathbf{0 . 2 7 6}$ & $\mathbf{0 . 0 0 4}$ & $\mathbf{0 . 4 9 3}$ & $<0.000$ \\
Nasal para-macular & -0.051 & 0.608 & $\mathbf{0 . 5 2 4}$ & $<\mathbf{0 . 0 0 0}$ \\
\hline Average peri- macular & $\mathbf{0 . 2 5 1}$ & $\mathbf{0 . 0 1 1}$ & $\mathbf{0 . 5 1 6}$ & $<\mathbf{0 . 0 0 0}$ \\
Superior peri -macular & 0.110 & 0.269 & $\mathbf{0 . 4 1 5}$ & $<\mathbf{0 . 0 0 0}$ \\
Temporal peri -macular & $\mathbf{0 . 3 5 6}$ & $\mathbf{0 . 0 0 0}$ & $\mathbf{0 . 4 4 2}$ & $<\mathbf{0 . 0 0 0}$ \\
Inferior peri -macular & $\mathbf{0 . 3 9 7}$ & $\mathbf{0 . 0 0 0}$ & $\mathbf{0 . 4 8 8}$ & $<\mathbf{0 . 0 0 0}$ \\
Nasal peri-macular & 0.054 & 0.588 & $\mathbf{0 . 5 2 7}$ & $<\mathbf{0 . 0 0 0}$ \\
\hline Overall & $\mathbf{0 . 5 0 3}$ & $<\mathbf{0 . 0 0 0}$ & $\mathbf{0 . 5 0 3}$ & $<\mathbf{0 . 0 0 0}$ \\
\hline
\end{tabular}

\section{Discussion}

The current study reports the onset of PVD was in the third decade of life but it did not show any difference in the degree of myopia. However, previous publications $[4,25]$ report the onset of PVD in high myopia (worse than $-8.00 \mathrm{D} \mathrm{SE}$ ) was in the third decade of life. This difference could be due to the methodology. We report the changes in foveal morphology and microvasculature in myopia and its correlation with various stages of PVD. In those with no PVD, high myopia patients had increased foveal thickness and macular cube volume as compared to low and moderate myopia. We also found that in those with no PVD, the FAZ

201 area became smaller as the degree of myopia increased. Previous studies have shown 202 conflicting results regarding foveal thickness and severity of myopia [26]. Samuel et al. [7] 203 reported results similar to ours, namely higher foveal thickness in high myopia. They also reported a correlation with the axial length, namely, longer axial lengths had higher foveal thickness. Similarly, Xie et al. [27] showed that the minimum and average foveal thickness were similar in emmetropia and low myopia groups but increased in high myopia groups.

207 However, Lim et al. [28] showed that the average macular retinal thickness did not 208 significantly vary between various degrees of myopia. The possible reason for discrepancies 209 in the association of myopia severity and foveal thickness could be due to the fact that these 
studies did not take into account the influence of PVD. We found that there was a reduction

211 in macular volume with degree of myopia. This result is similar to that of Hwang et al. [29]

212 who found that macular volume was lower in very-high myopia. This could be due to

213 progressive thinning of the para- and peri- foveal thickness with increasing myopia. Zhao et

214 al. [16] reported that the para- and peri-foveal thickness were negatively correlated with the

215 axial length and positively associated with the SE.

216 Gołębiewska et al. [30] studied the foveal microvasculature and found that the FAZ area was

217 larger in myopic eyes than in the control group. However, studies by Leng et al. [31] and

218 Ucaket al. [32] showed no difference in FAZ area in both high and low myopic eyes. The

219 custom developed automated algorithm [22] used in this study could detect boundaries with

220 an error of $1.99 \%$ compared to the manufacturer's inbuilt algorithm with $6.42 \%$ error

221 excluding orientation. This thresholding method dilates the irrelevant part of the image

222 without distorting the actual FAZ boundary and reduces the number of false positives. In this

223 study we found a smaller FAZ area in high myopia in subjects with IPVD attached at the

224 macula.

225 Kumagai et al. [33] described the changes in OCT characteristics after an asymptomatic PVD

226 and showed a thinning of the central foveal thickness, deepening of the pit, and widening of

227 the foveal floor after PVD. The mechanism by which the PVD alters the foveal shape and

228 regional macular thickness is not understood. Possible mechanisms may include the release

229 of the tractional force on the fovea by the peri-foveal IPVD. An anterior centrifugal traction

230 could cause a thickening of the fovea, and the release of this traction would then presumably

231 reduce the foveal thickness. Likewise, mechanical forces might explain the smaller FAZ in high

232 myopia with IPVD attached at macula. These results come from a relatively small sample size

233 and hence should be considered as preliminary results. The clinical significance of these

234 results, namely that there is an increasing trend of partial PVD has definite clinical implications

235 and need to be further explored. 
Since the current study was retrospective, we had very few cases with axial length data. The

237 best way to classify myopia should be based on the corneal-axial ratio or at least by axial 238 length. Sampson et al. [20] reported that the FAZ area is affected by its axial magnification 239 during the image acquisition. This can be rectified by correcting for image magnification 240 induced by the axial length variation [34]. In cases of high myopia (SE -6.00 to -10.00D) or very 241 high myopia (SE >-10.00 D) of our sample, none of the cases had a corneal curvature $>44.00 \mathrm{D}$. 242 Likewise, no eyes had cataractous changes and all the subjects had axial myopia. The second 243 shortcoming was the low sample size of the various myopic sub-groups. We hope to further 244 expand on the datasets as well as pathological conditions in future research.

\section{Conclusions}

246 In summary, we report characteristic retinal changes in high and very high myopic eyes.

247 An increasing trend of partial PVD is seen in eyes classified as having high and very high 248 myopia. An asymmetric PVD in location was observed and the most commonly involved 249 quadrant was the inferior quadrant. A statistically significant alteration was observed in foveal 250 volume and the FAZ circularity index. However, our data did not show any relationship 251 between PVD and macular thickness and foveal microvascular parameters in high myopic 252 eyes.

\section{List of abbreviations}

255 PVD: Posterior vitreous detachment, SD-OCT: Spectral-domain optical coherence 256 tomography, SE: Spherical equivalent, CRT: Central macular thickness, FAZ: Foveal 257 microvascular zone dimensions, OCTA: Optical coherence tomography angiography, OCT: 258 Optical coherence tomography, MT: macular thickness, BCVA: Best corrected visual acuity, 259 MCV: Macular cube volume, D: Diopter, LM: Low myopia, MM: Moderate myopia, HM: High 260 myopia, VHM: Very high myopia. 
Ethics approval and consent to participate

This retrospectives study was approved by the Institutional Review Board of the Vision

\section{Consent for publication}

Not applicable

\section{Availability of data and materials}

273 All the clinical data and materials supporting the manuscript are maintained in our Hospital.

\section{Competing interests}

The authors declare that they have no competing interests.

\section{Funding}

279 None

\section{Authors' Contribution}

Concept and design: JJB, RR and VL; Software Development and validation: AA and VL; Data

283 acquisition: JJB; Data analysis / interpretation: JJB and VL; Draft the manuscript: JJB; Critical

284 revision of the manuscript: VL and RR; Supervision: VL and RR. All authors read and approved 285 the final manuscript. 
289 The authors would like to thank the subjects for agreeing to report the clinical data. This work 290 was partly supported by a DISCOVERY Grant from the Natural Sciences and Engineering 291 Research Council of Canada to V.L.

\section{Authors' information}

JJB: Department of Optometry, Sankara Nethralaya, Medical Research Foundation, Chennai, India. AA: Department of Chemical Engineering, Indian Institute of Technology, Kanpur, India.

296 Currently working with Goldman Sachs Services Private limited, India. RR: Shri Bhagwan 297 Mahavir Vitreoretinal Services, Sankara Nethralaya, Medical Research Foundation, Chennai, 298 India. VL: Theoretical and Experimental Epistemology Lab, School of Optometry and Vision 299 Science, Departments of Physics, Electrical and Computer Engineering and the Systems Design 300 Engineering, University of Waterloo, Waterloo, Ontario, Canada.

\section{References}

1. Sebag J: The vitreous: structure, function, and pathobiology. 1989; Springer Verlag New York.

2. Yıldııım Ş, Menteş J, Barış M: Vitreomacular Traction and Outer Retinal Structural Changes. Turkish J Ophthalmol. 2019;49(2):109-113

3. Stalmans P, Jay SD, Peter KK, Jeffrey SH, Pravin UD, Arnd G, Sebag J, Julia AH: OCTBased Interpretation of the vitreomacular interface and indications for pharmacologic vitreolysis. Retina. 2013;33(10):2003-2011.

4. Itakura H, Kishi S, Li D, Nitta K, Akiyama H: Vitreous changes in high myopia observed by swept-source optical coherence tomography. Investig Ophthalmol Vis Sci. 2014;55(3):1447-52. 
5. Kakehashi A, Takezawa M Akiba J: Classification of Posterior Vitreous Detachment. Clin Ophthalmol. 2014;8:1-10.

6. Mirza RG, Johnson MW, Jampol LM: Optical Coherence Tomography Use in Evaluation of the vitreoretinal interface: A review. Surv Ophthalmol. 2007;52(4):397-421.

7. Samuel NE, Krishnagopal S: Foveal and Macular Thickness Evaluation by Spectral OCT SLO and its Relation with Axial Length in Various Degree of Myopia. J Clin Diagnostic Res: JCDR 2015;9(3):1-4.

8. Koh VT, Nah GK, Chang L, Yang AH, Lin ST, Ohno-Matsui K, Wong TY, Saw SM: Pathologic changes in highly myopic eyes of young males in Singapore. Ann. Acad. Med. Singapore. 2013;42(5):216-224.

9. Tsujikawa A, Kikuchi M, Ishida K, Nonaka A, Yamashiro K, Kurimoto, Y: Fellow eye of patients with retinal detachment associated with macular hole and bilateral high myopia. Clin Exp Ophthalmol. 2006;34(5):430-433.

10. Zhao $\mathrm{M}, \mathrm{Wu} \mathrm{Q}, \mathrm{Hu} \mathrm{P}$, Jia L: Macular thickness assessed with optical coherence tomography in young Chinese myopic patients. J Ophthalmol. 2015;doi:10.1155/2015/715798.

11. Akiba J: Prevalence of posterior vitreous detachment in high myopia. Ophthalmology. 1993;100(9):1384-1388.

12. Bond-Taylor $M$, Jakobsson $G$, Zetterberg $M$ : Posterior vitreous detachmentprevalence of and risk factors for retinal tears. Clin. Ophthalmol. 2017;11: 1689-1695. 13. Shen Z, Duan X, Wang F, Wang N, Peng Y, Liu DT, Peng X, Li S, Liang Y: Prevalence and risk factors of posterior vitreous detachment in a Chinese adult population: the Handan eye study. BMC ophthalmol. 2013;13(1):1-6.

14. AttaAllah HR, Omar IA, Abdelhalim AS: Assessment of Posterior Segment Using Spectral Domain OCT in Highly Myopic Eyes. The open ophthalmol J. 2017;11:334345. 

and Axial Length in Myopic Eyes. BioMed Res. Int 2019;2019:1-7.

16. Zhao Z, Zhou X, Jiang C, Sun X: Effects of myopia on different areas and layers of the macula: a Fourier-domain optical coherence tomography study of a Chinese cohort. BMC Ophthalmol. 2015;15(1):1-7.

17. Abouhussein MA Gomaa AR: Optical coherence tomography findings in high myopia. Egypt Retina J. 2013;1(2):13-17.

18. Ito $\mathrm{Y}$, Terasaki H, Suzuki T, Kojima T, Mori M, Ishikawa K, Miyake Y: Mapping posterior vitreous detachment by optical coherence tomography in eyes with idiopathic macular hole. Am J Ophthalmol. 2003;135(3):351-355.

19. Xu LT, Punjabi OS, Shao J, Ehlers JP, Srivastava SK, Singh RP Kaiser PK: Frequency of vitreomacular interface disease in patients presenting to a tertiary care institution. Ophthalmic Surg Lasers Imaging Retin. 2014;45(6):517-20.

20. Sampson DM, Gong P, An D, Menghini M, Hansen A, Mackey DA, Sampson DD, Chen FK: Axial length variation impacts on superficial retinal vessel density and foveal avascular zone area measurements using optical coherence tomography angiography. Investig Ophthalmol Vis Sci. 2017;58(7):3065-3072.

21. Dhakal R, Goud A, Narayanan R, Verkicharla PK: Patterns of posterior ocular complications in myopic eyes of Indian population. Sci rep. 2018;8(1):1-6.

22. Agarwal A, Balaji JJ, Lakshminarayanan V: A new technique for estimating the foveal avascular zone dimensions. in Ophthalmic Technologies XXX, International Society for Optics and Photonics. 2020; 11218R:2020. doi:10.1117/12.2543906. zone in diabetic retinopathy, high myopia, and normal fundus images. in Ophthalmic Technologies XXX, International Society for Optics and Photonics. 2020; 112180: 2020. doi:10.1117/12.2544817 

F, Gillies M, Staurenghi G: Normative data for retinal-layer thickness maps generated by spectral-domain OCT in a white population. Ophthalmol Retina. 2018;2(8):808-15.

25. Morita $H$, Funata $M$, Tokoro $T$ : A clinical study of the development of posterior vitreous detachment in high myopia. Retina. 1995;15(2): 117-124.

26. Lim, Hyun-Taek, Chun BY: Comparison of OCT Measurements between High Myopic and Low Myopic Children, Optom Vis Sci. 2013;90(12):1473-1478. major biometric parameters of the eye: a retrospective clinical study. Optom Vis Sci.

29. Hwang YH, Kim YY: Macular thickness and volume of myopic eyes measured using spectral-domain optical coherence tomography. Clin Exp Optom. 2012;95(5):492-498.

30. Gołębiewska J, Biała-Gosek K, Czeszyk A, Hautz W: Optical coherence tomography angiography of superficial retinal vessel density and foveal avascular zone in myopic children. PloS one. 2019;14(7):e0219785. doi:10.1371/journal.pone.0219785.

31. Leng Y, Tam EK, Falavarjani KG, Tsui I: Effect of age and myopia on retinal microvasculature. Ophthalmic Surg. Lasers Imaging Retin. 2018;49(12):925-931.

32. Ucak T, Icel E, Yilmaz H, Karakurt Y, Tasli G, Ugurlu A, Bozkurt E: Alterations in optical coherence tomography angiography findings in patients with high myopia. Eye. 2020;34(6):1-7.

33. Kumagai K, Hangai $M$, Larson E, Ogino $N$ : Vitreoretinal interface and foveal deformation in asymptomatic fellow eyes of patients with unilateral macular holes. 

Systematic Review. JAMA Ophthalmol. 2020;138(1):86-94. 


\section{Figures}

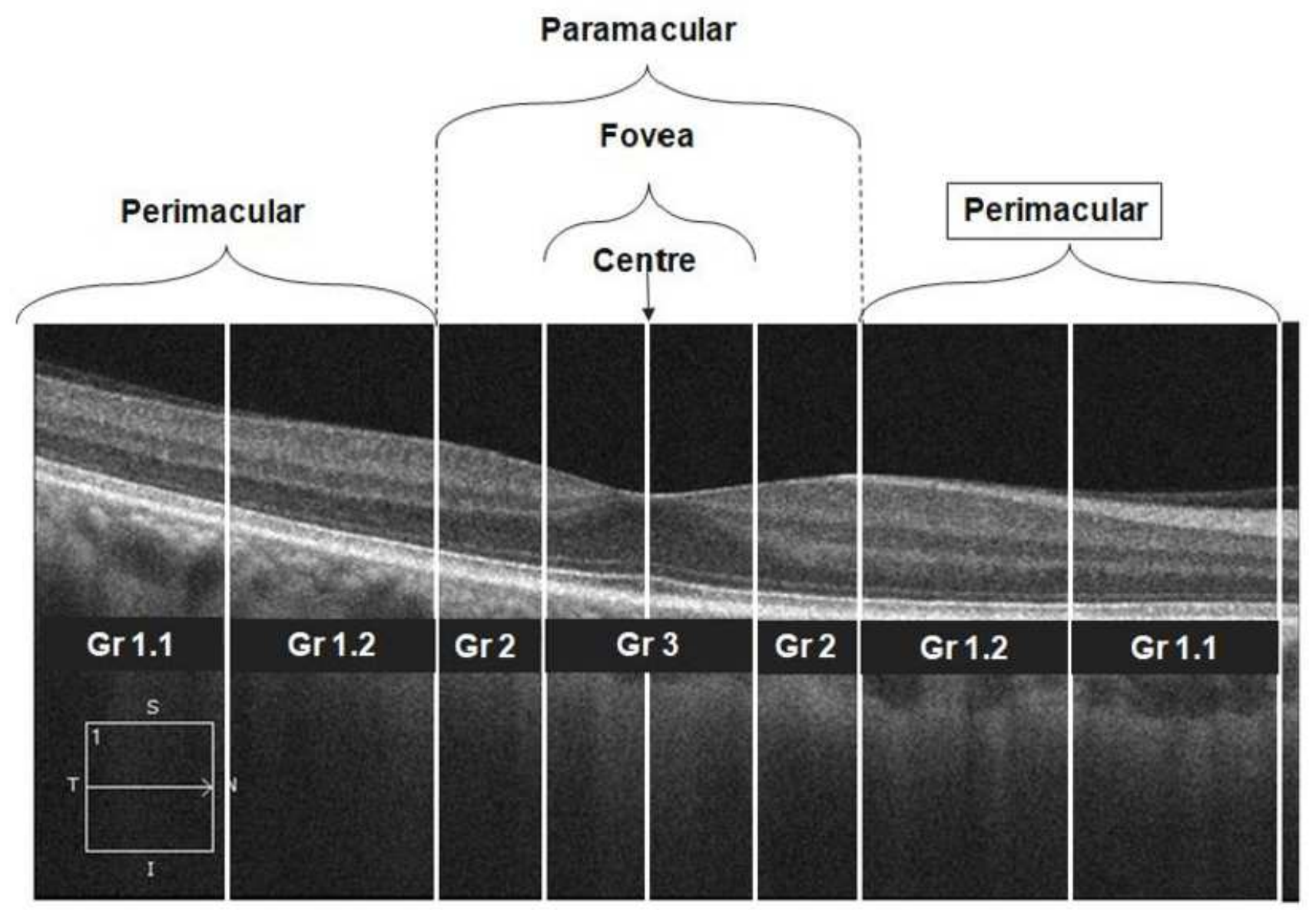

Figure 1

An example and a schematic diagram of a SD-OCT horizontal radial scan image. The PVD classification based on the PVD location. The above picture shows the PVD extended till parimacular area with grade 1.2. 

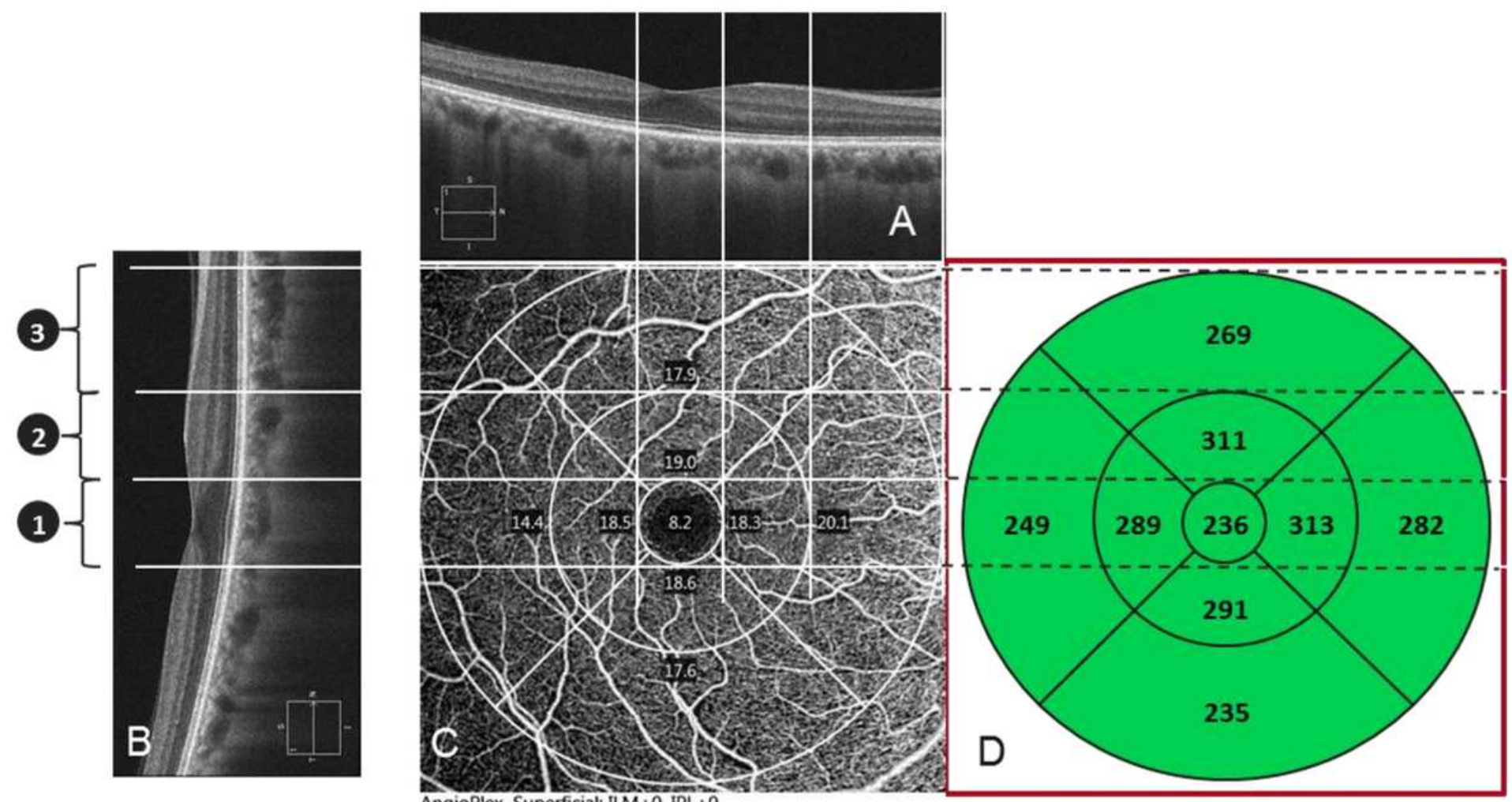

AngioPlex Superficial: ILM+0, IPL+0

Figure 2

A relationship between PVD location and the corresponding location macular thickness and angioplex image using the ETDRS method. A \& B) a radial scan of a horizontal and vertical scan. The numbers 1, 2 \& 3 indicating the macular anatomical structures 1) fovea, 2) paramacular and 3) perimacular area. C) An en-face image of macular and FAZ with ETDRS segmentation D) the ETDRS macular thickness (in microns) to the corresponding area of the fovea, para, and peri macular regions. 

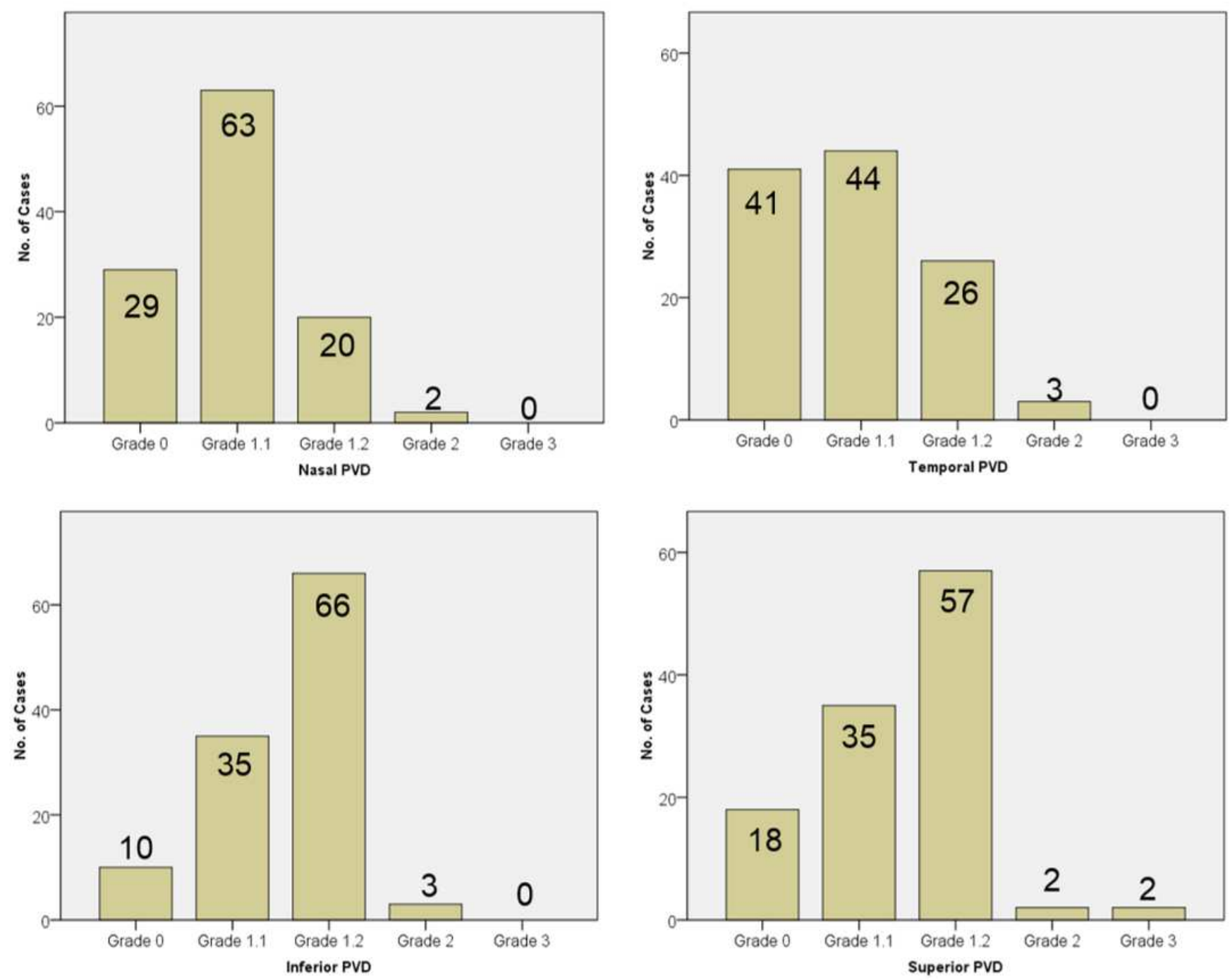

Figure 3

Distribution of PVD in all four quadrants. 


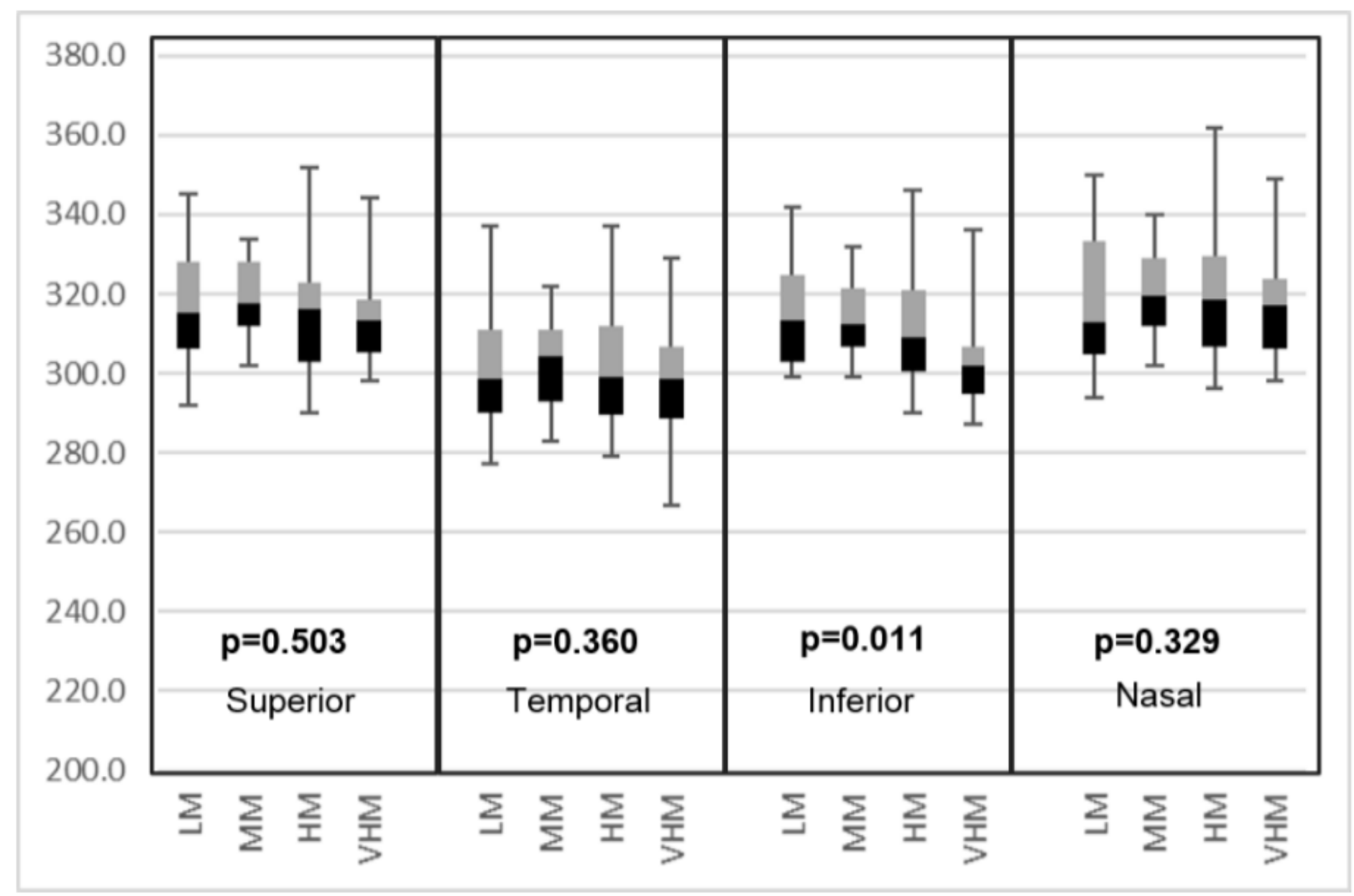

Figure 4

A box-plot between para-macular thickness in four quadrants and degree of myopia LM: Low myopia, MM: Moderate myopia, HM: High myopia VHM: Very high myopia 


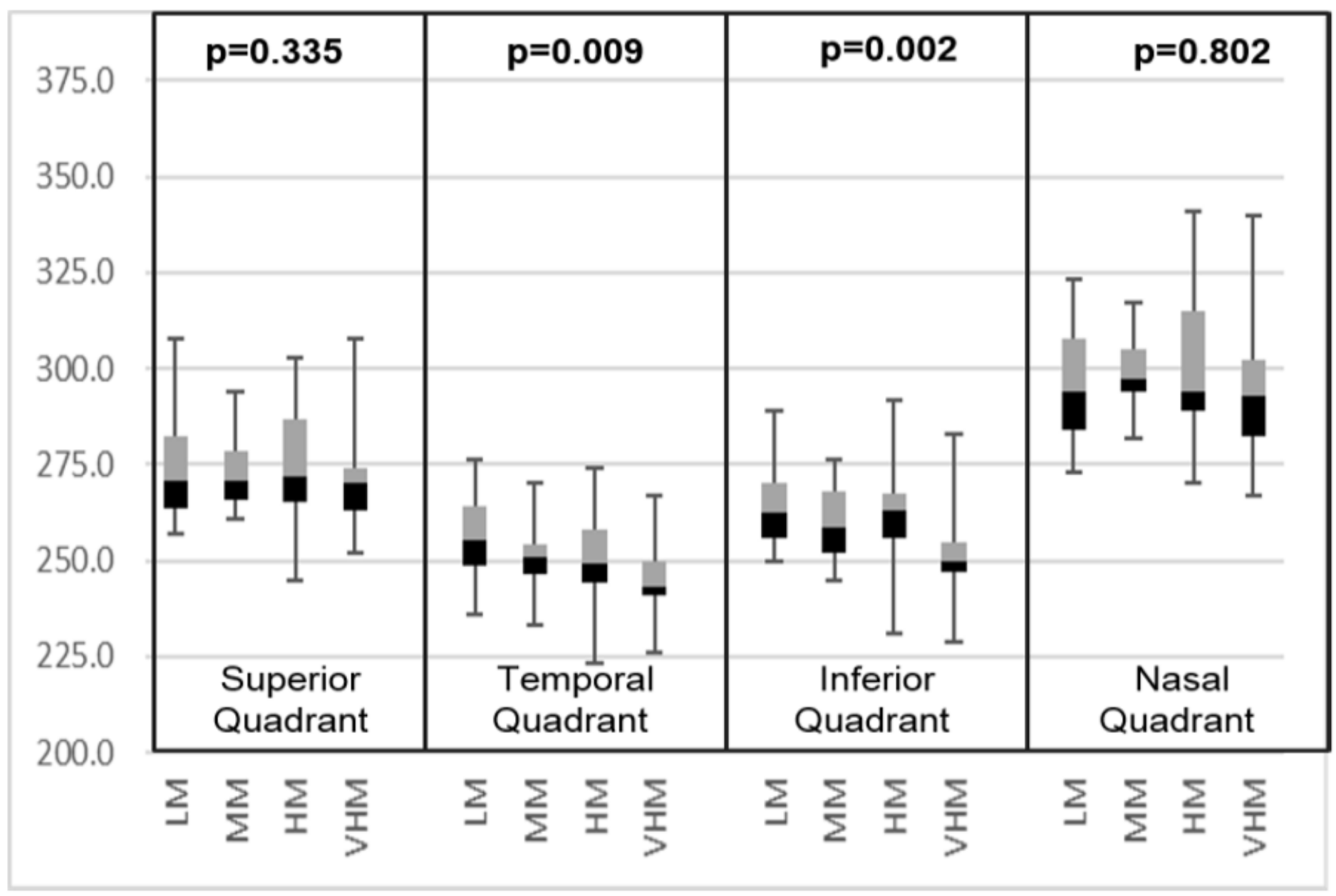

Figure 5

A box-plot of Peri-macular thickness in all the four quadrants and degree of myopia 


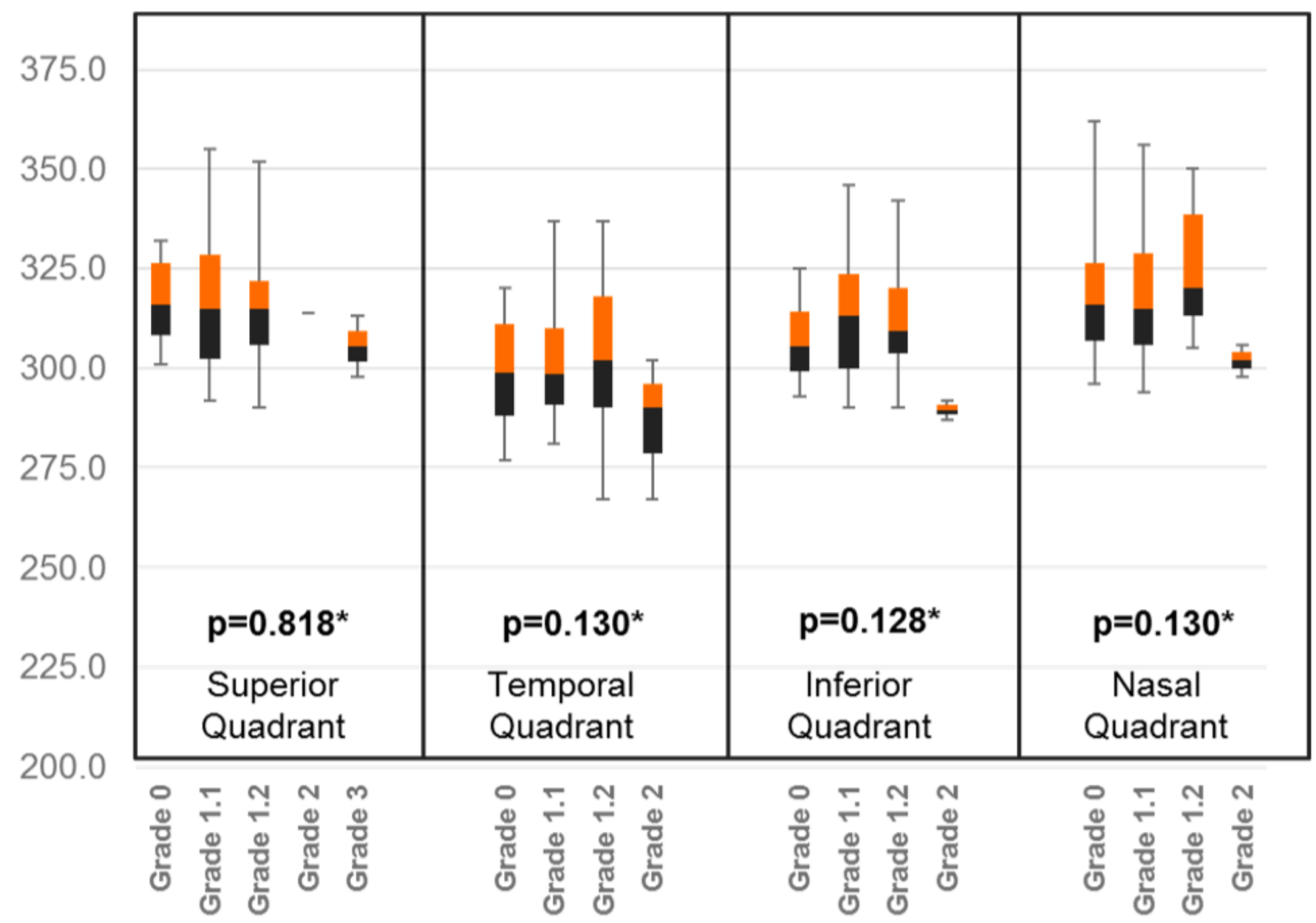

Figure 6

Relationship between paramacular vessel density \& PVD stages 


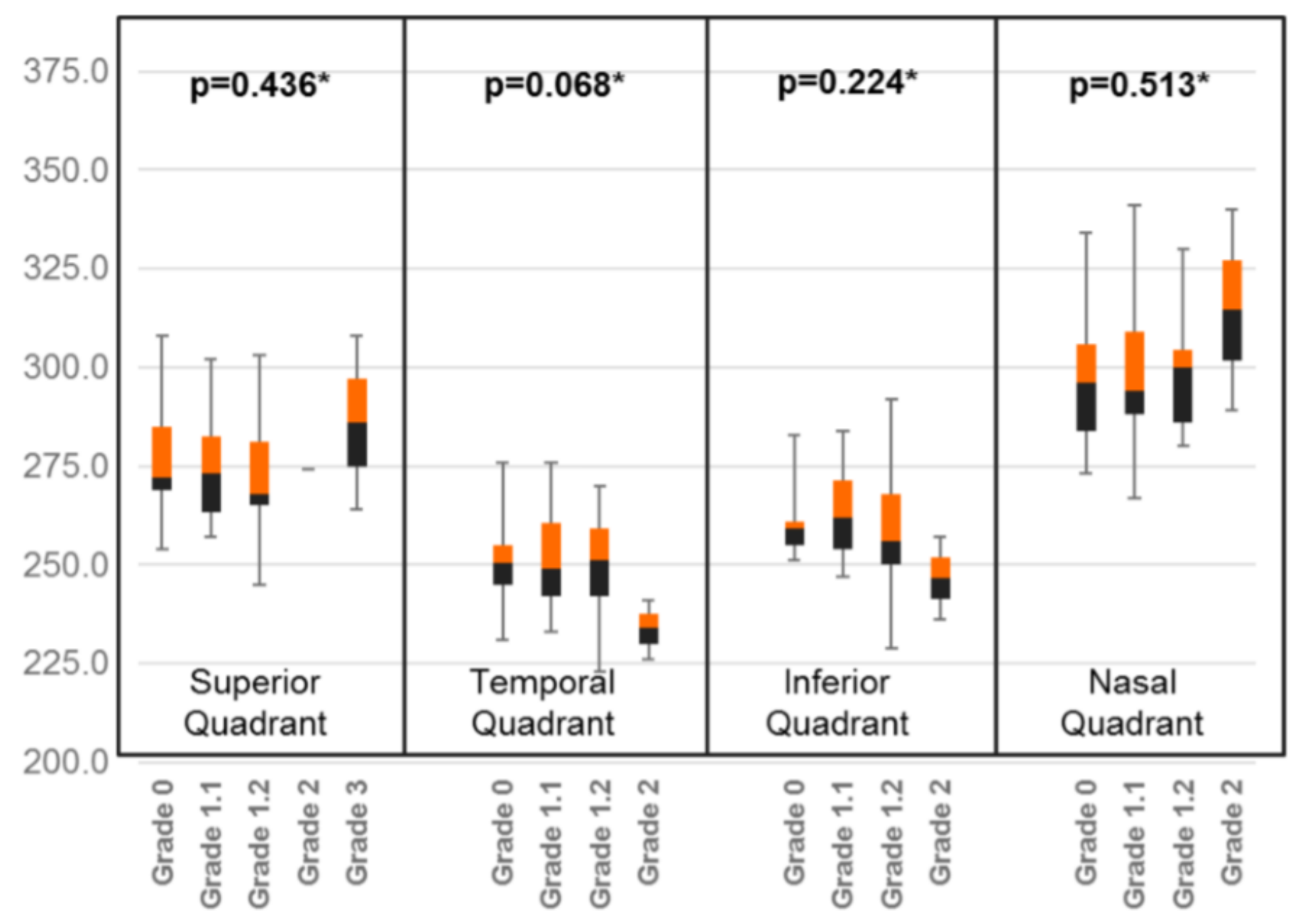

Figure 7

Relationship between peri-macular vessel density \& PVD stages 